gap> $\mathrm{g}:=$ SymmetricGroup ( 4 );

$\operatorname{Sym}([1 \ldots 4])$

i5 : betti $(t$, Weights $\Rightarrow\{1$, gap $\}$

false

01234 gap> tblmod2:= CharacterTable( tbl, 2);

05 = total: 1413144 BrauerTable $(\operatorname{Sym}([1 \ldots 4]), 2$ )

1: . 2242 gap> tblmod2 = CharacterTable $(t b l, 2)$;

Journal of Software for

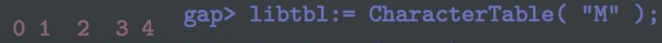

$06=$ total: 1413144 CharacterTable( "M")

fail $\quad r i n g ~ r 1=32003,(x, y, z)$, ds;

gap> CharacterTable( "Symmetric", 4 ) ; int a,b,c,t=11,5,3,0;

BettiTally

CharacterTable( "Sym(4)")

gap> ComputedBrauerTables ( tbl );

$\begin{array}{lllll}0 & 1 & 2 & 3 & 4\end{array}$

[ poly $f=x^{\wedge} a+y^{\wedge} b+z^{\wedge}(3 * c)+x^{\wedge}(c+2) * y^{\wedge}(c-1)+x^{\wedge}$ $\mathrm{x}^{\sim}(\mathrm{c}-2) * \mathrm{y}^{\wedge} \mathrm{c} *\left(\mathrm{y}^{\sim} 2+\mathrm{t} * \mathrm{x}\right)^{\sim}-2$;

o7 = total: 1413144

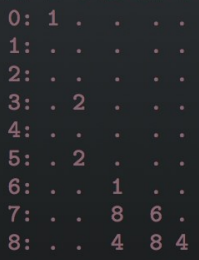

o7 : BettiTally

i8 : peek t1

$08=$ BettiTally $\{(0,\{0,0\}, 0) \Rightarrow 1\}$

$(1,\{2,2\}, 4) \Rightarrow 2$

$(1,\{3,3\}, 6) \Rightarrow 2$

(2, $\{3,7\}, 10) \Rightarrow 2$

$(2,\{4,4\}, 8) \Rightarrow 1$

(2, $\{4,5\}, 9) \Rightarrow 4$

$(2,\{5,4\}, 9) \Rightarrow 4$

(2, $\{7,3\}, 10) \Rightarrow 2$

$(3,\{4,7\}, 11) \Rightarrow 4$

Stronglitis stable ideals and Hilbert polynomials

$(4,\{5,7\}, 12) \Rightarrow 2$

$(4,\{7,5\}$, D 2 AVPDE ALBERELLI AND PAOLO LELLA

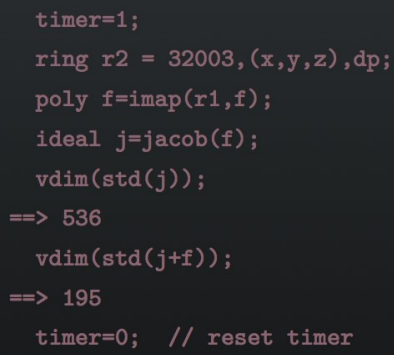




\title{
Strongly stable ideals and Hilbert polynomials
}

\author{
Davide Alberelli AND PaOlo Lella
}

\begin{abstract}
The StronglyStableIdeals.m2 package for Macaulay2 provides a method to compute all saturated strongly stable ideals in a given polynomial ring with a fixed Hilbert polynomial. A description of the main method and auxiliary tools is given.
\end{abstract}

INTRODUCTION. Strongly stable ideals are a key tool in commutative algebra and algebraic geometry. These ideals have nice combinatorial properties that make them well suited for both theoretical and computational applications. In the case of polynomial rings with coefficients in a field of characteristic zero, the notion of strongly stable ideals coincides with the notion of Borel-fixed ideals. Such ideals are fixed by the action of the Borel subgroup of triangular matrices and play a special role in the theory of Gröbner bases because initial ideals in generic coordinates are of this type [Galligo 1974].

In the context of parameter spaces of algebraic varieties, Galligo's theorem says that each component and each intersection of components of a Hilbert scheme contains at least one point corresponding to a scheme defined by a Borel-fixed ideal. Hence, these ideals are distributed throughout the Hilbert scheme and can be used to study its local structure. To this end, in recent years several authors [Lella and Roggero 2011; 2016; Cioffi and Roggero 2011; Bertone et al. 2013a; 2017a; 2017b] developed algorithmic methods based on the use of strongly stable ideals to construct flat families corresponding to special loci of the Hilbert scheme. In particular, a new open cover of the Hilbert scheme has been defined using strongly stable ideals and the action of the projective linear group [Bertone et al. 2013b; Brachat et al. 2016]. In this construction, the list of all points corresponding to Borel-fixed ideals in a given Hilbert scheme is needed. The main feature of the package StronglyStableIdeals.m2 is a method to compute this set of points, i.e., the list of all saturated strongly stable ideals in a polynomial ring with a given

The second author is a member of GNSAGA .

MSC2010: primary 13P10; secondary 13P99.

Keywords: strongly stable ideal, Borel-fixed ideal, Hilbert polynomial, Gotzmann number, Hilbert scheme.

StronglyStableIdeals.m2 version 1.1 
Hilbert polynomial. The method has been theoretically introduced in [Cioffi et al. 2011] and improved in [Lella 2012]. Several other tools are developed and presented in the current paper.

1. Strongly Stable ideals. Let us denote by $\mathbb{R}[\boldsymbol{x}]$ the polynomial ring in $n+1$ variables $\mathbb{K}\left[x_{0}, \ldots, x_{n}\right]$ with coefficients in a field $\mathbb{K}$. We assume that $x_{0}>x_{1}>\cdots>x_{n}$. We use the multi-index notation to describe monomials, i.e., $\boldsymbol{x}^{\alpha}:=x_{0}^{\alpha_{0}} \cdots x_{n}^{\alpha_{n}}$ for every $\boldsymbol{\alpha}=\left(\alpha_{0}, \ldots, \alpha_{n}\right) \in \mathbb{Z}_{\geqslant 0}^{n+1}$, and we denote by $\mathbb{T}_{n, s}$ the set of monomials of $\mathbb{K}[\boldsymbol{x}]$ of degree $s$. For any monomial $\boldsymbol{x}^{\alpha}$, we denote by $\min \boldsymbol{x}^{\alpha}$ and $\max \boldsymbol{x}^{\alpha}$ the indices of the minimal and maximal variable dividing $\boldsymbol{x}^{\alpha}$.

Following [Green 2010], increasing and decreasing elementary moves are defined as the multiplications

$$
\mathrm{e}_{i}^{+}\left(x^{\alpha}\right):=\frac{x_{i-1}}{x_{i}} \cdot x^{\alpha}, \quad i>0, \quad \text { and } \quad \mathrm{e}_{j}^{-}\left(x^{\alpha}\right):=\frac{x_{j+1}}{x_{j}} \cdot x^{\alpha}, \quad j<n .
$$

We say that an elementary move $\mathrm{e}_{i}^{+/-}$is admissible for a monomial $\boldsymbol{x}^{\alpha}$ if $\alpha_{i}>0$, that is, $\mathrm{e}_{i}^{+/-}\left(\boldsymbol{x}^{\alpha}\right)$ is a monomial of $\mathbb{K}[\boldsymbol{x}]$.

Definition 1.1. An ideal $I \subset \mathbb{K}[x]$ is called strongly stable if

(i) $I$ is a monomial ideal;

(ii) for every $\boldsymbol{x}^{\alpha} \in I$ and for every admissible increasing move $\mathrm{e}_{i}^{+}$, the monomial $\mathrm{e}_{i}^{+}\left(\boldsymbol{x}^{\alpha}\right)$ is contained in $I$.

We recall that a strongly stable ideal is a Borel-fixed ideal. We now summarize some properties holding in general for Borel-fixed ideals and useful in this context.

Proposition 1.2 [Green 2010, Section 2]. Let $I \subset \mathbb{K}[\boldsymbol{x}]$ be a strongly stable ideal.

(i) The regularity of I is equal to the maximal degree of a generator.

(ii) Let $\mathfrak{m}$ be the irrelevant ideal of $\mathbb{K}[x]$. Then, $(I: \mathfrak{m})=\left(I: x_{n}\right)$, so that the ideal $I$ is saturated if no generator involves the last variable $x_{n}$.

(iii) The last variable $x_{n}$ is a regular element for I, i.e., the multiplication by $x_{n}$ induces the short exact sequence

$$
0 \longrightarrow \frac{\mathbb{K}[\boldsymbol{x}]}{I}(t-1) \stackrel{\cdot x_{n}}{\longrightarrow} \frac{\mathbb{K}[\boldsymbol{x}]}{I}(t) \longrightarrow \frac{\mathbb{K}[\boldsymbol{x}]}{\left(x_{n}, I\right)}(t) \longrightarrow 0
$$

2. Hilbert polynomials. The Hilbert polynomial $p(t)$ of a homogeneous ideal $I \subset \mathbb{K}[\boldsymbol{x}]$ is the numerical polynomial such that for $s$ sufficiently large

$$
\operatorname{dim}_{\mathbb{K}}\left(\frac{\mathbb{K}[\boldsymbol{x}]}{I}\right)_{s}=\operatorname{dim}_{\mathbb{}}\left(\frac{\mathbb{K}[\boldsymbol{x}]_{s}}{I_{s}}\right)=\left(\begin{array}{c}
n+s \\
n
\end{array}\right)-\operatorname{dim}_{\mathbb{K}} I_{s}=p(s) .
$$


Obviously, not every numerical polynomial is a Hilbert polynomial of some homogeneous ideal. Those being Hilbert polynomials have been completely described by Gotzmann [1978].

Gotzmann's decomposition. A numerical polynomial $p(t) \in \mathbb{Q}[t]$ is a Hilbert polynomial if, and only if, it can be written as

$$
p(t)=\left(\begin{array}{c}
n+a_{1} \\
a_{1}
\end{array}\right)+\left(\begin{array}{c}
n+a_{2}-1 \\
a_{2}
\end{array}\right)+\cdots+\left(\begin{array}{c}
n+a_{r}-(r-1) \\
a_{r}
\end{array}\right), \quad a_{1} \geqslant \cdots \geqslant a_{r} \geqslant 0 .
$$

This decomposition is strictly related to Macaulay's decomposition

$$
p(t)=\sum_{k=0}^{d}\left[\left(\begin{array}{l}
t+k \\
k+1
\end{array}\right)-\left(\begin{array}{c}
t+k-m_{k} \\
k+1
\end{array}\right)\right],
$$

where $d=\operatorname{deg} p(t)$. For all $n \geqslant d+1$ the saturated lexicographic ideal within $\mathbb{K}\left[x_{0}, \ldots, x_{n}\right]$ with Hilbert polynomial $p(t)$ is

$$
\left(x_{0}, \ldots, x_{n-d-2}, x_{n-d-1}^{b_{d}+1}, x_{n-d-1}^{b_{d}} x_{n-d}^{b_{d-1}+1}, \ldots, x_{n-d-1}^{b_{d}} x_{n-d}^{b_{d-1}} \cdots x_{n-1}^{b_{0}}\right),
$$

where

$b_{d}=\#\left\{a_{j} \mid a_{j}=d\right\}=m_{d} \quad$ and $\quad b_{k}=\#\left\{a_{j} \mid a_{j}=k\right\}=m_{k}-m_{k+1}, \quad 0 \leqslant k<d$.

The description of the lexicographic ideal in terms of Gotzmann's decomposition gives an insight to the following theorem.

Gotzmann's regularity theorem. The regularity of a saturated ideal $I \subset \mathbb{K}[\boldsymbol{x}]$ with Hilbert polynomial $p(t)$ is at most $r$, where $r$ is the number of terms in the decomposition (1) and it is called the Gotzmann number of $p(t)$.

Example 2.1. The package StronglyStableIdeals.m2 provides the method isHilbertPolynomial to determine if a numerical polynomial is a Hilbert polynomial.

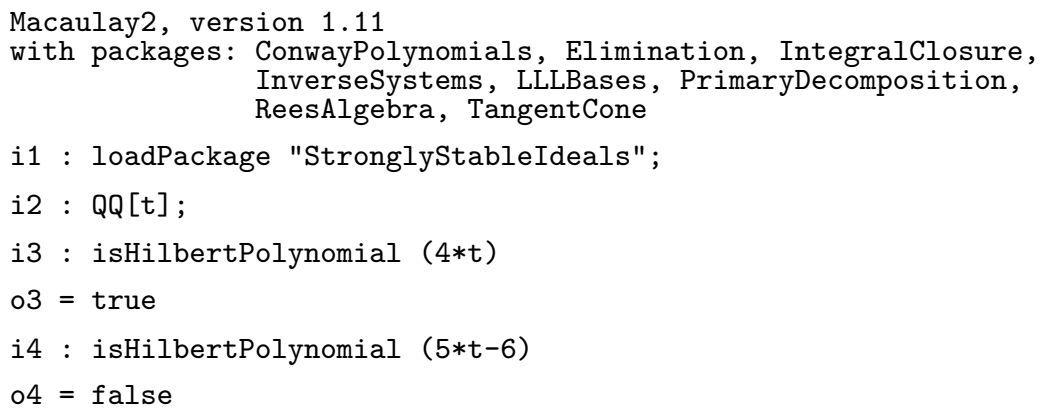

Gotzmann's and Macaulay's decompositions of a Hilbert polynomial can be computed using gotzmannDecomposition and macaulayDecomposition. These 
methods return the list of terms in the decompositions. The summand $\left(\begin{array}{c}t+e \\ c\end{array}\right)$ is constructed with the command projectiveHilbertPolynomial (c, c-e).

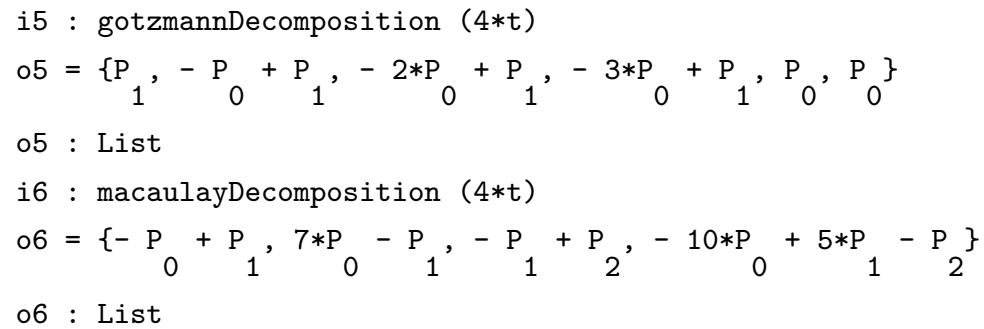

Finally, the saturated lexicographic ideal $L$ with Hilbert polynomial $p(t)$ in the polynomial ring $\mathbb{K}[\boldsymbol{x}]$ can be computed with the method lexIdeal and its regularity is equal to the Gotzmann number of $p(t)$.

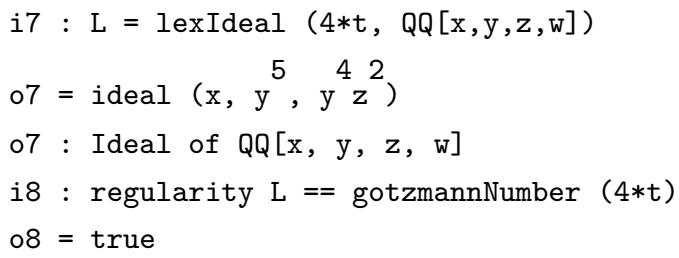

3. The MAIN ALGORITHM. In this section, we outline the strategy of the main algorithm. This algorithm was firstly described in [Cioffi et al. 2011] and then optimized in [Lella 2012]. The same problem has been previously discussed in [Reeves 1992] and an alternative algorithm was later presented in [Moore and Nagel 2014].

We need to relate the properties of a strongly stable ideal with its Hilbert polynomial. If $I$ is a strongly stable ideal, for each $s \in \mathbb{N}$ the monomial basis of the homogeneous piece $I_{s}$ of the ideal is a subset of $\mathbb{T}_{n, s}$ closed by increasing elementary moves. We call Borel sets such subsets of $\mathbb{T}_{n, s}$ (see Figure 1 for an example). Proposition 1.2(i) implies that the monomial basis of $I_{s}$ for a saturated strongly stable ideal $I \subset \mathbb{K}[\boldsymbol{x}]$ with Hilbert polynomial $p(t)$ and regularity at most $s$ is a Borel set with $q(s):=\left(\begin{array}{c}n+s \\ n\end{array}\right)-p(s)$ elements. Thus, we consider the map

$$
\left\{\begin{array}{c}
\text { saturated strongly stable ideals in } \mathbb{K}[\boldsymbol{x}] \text { with } \\
\text { Hilbert polynomial } p(t) \text { and regularity } \leqslant s
\end{array}\right\} \hookrightarrow\left\{\begin{array}{c}
\text { Borel sets of } \mathbb{T}_{n, s} \\
\text { with } q(s) \text { elements }
\end{array}\right\} .
$$

Moreover, Gotzmann's regularity theorem suggests considering $s$ equal to the Gotzmann number of $p(t)$ to determine all saturated strongly stable ideals with Hilbert polynomial $p(t)$. Obviously, there are many Borel sets in $\mathbb{T}_{n, s}$ with $q(s)$ elements not corresponding to an ideal with Hilbert polynomial $p(t)$. To identify the image of the previous map, we recall a definition and a proposition by Mall. 

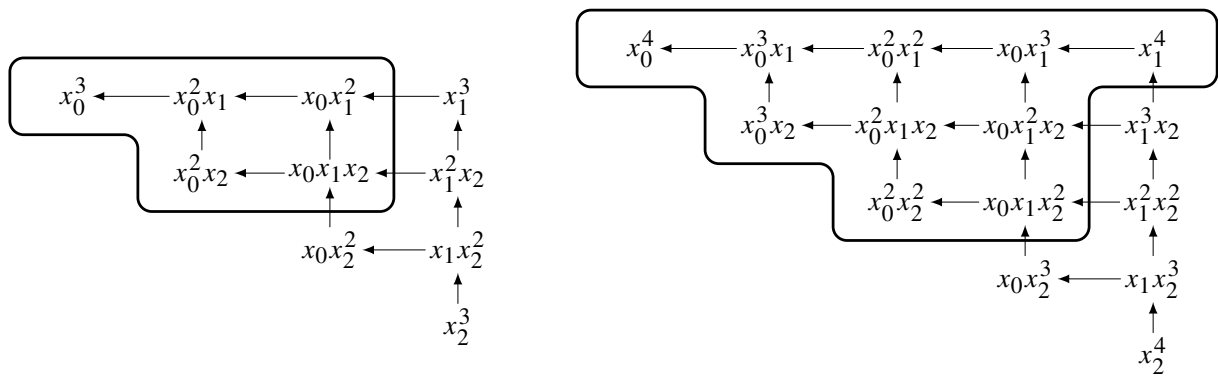

Figure 1. The Borel sets defined in $\mathbb{T}_{2,3}$ and $\mathbb{T}_{2,4}$ by the ideal $\left(x_{0}^{2}, x_{0} x_{1}, x_{1}^{4}\right) \subset \mathbb{K}\left[x_{0}, x_{1}, x_{2}\right]$.

Definition 3.1 [Mall 1997, Definition 2.7]. Let $B \subset \mathbb{T}_{n, s}$ be a Borel set. The set $B^{(i)}:=\left\{x^{\alpha} \in B \mid \min x^{\alpha}=n-i\right\}$ is called the $i$-growth class of $B$. The sequence $\operatorname{gv}(B):=\left(\left|B^{(0)}\right|, \ldots,\left|B^{(n)}\right|\right)$ is called the growth vector of $B$.

Proposition 3.2 [Mall 1997, Proposition 3.2]. Let $I \subset \mathbb{K}[\boldsymbol{x}]$ be a strongly stable ideal generated by the monomials of a Borel set $B \subset \mathbb{T}_{n, s}$ and let $p(t)$ be its Hilbert polynomial. Then,

$$
p(t)=\left(\begin{array}{c}
n+t \\
n
\end{array}\right)-\sum_{k=0}^{n}\left|B^{(k)}\right|\left(\begin{array}{c}
k+t-s \\
k
\end{array}\right), \quad \text { for all } t \geqslant s .
$$

We can use this result to determine the growth vector of a Borel set $B \subset \mathbb{T}_{n, s}$ starting from the Hilbert polynomial. The $i$-th difference polynomial of $p(t)$ is

$$
\left(\Delta^{i} p\right)(t)=\left(\Delta^{i-1} p\right)(t)-\left(\Delta^{i-1} p\right)(t-1)=\left(\begin{array}{c}
n+t-i \\
n-i
\end{array}\right)-\sum_{k=i}^{n}\left|B^{(k)}\right|\left(\begin{array}{c}
k+t-s-i \\
k-i
\end{array}\right) .
$$

Evaluating these identities at $t=s$, we obtain the linear system

$$
\left\{\begin{array}{l}
\sum_{k=0}^{n}\left|B^{(k)}\right|=\left(\begin{array}{c}
n+s \\
n
\end{array}\right)-p(s), \\
\vdots \\
\sum_{k=i}^{n}\left|B^{(k)}\right|=\left(\begin{array}{c}
n+s-i \\
n-i
\end{array}\right)-\left(\Delta^{i} p\right)(s), \\
\vdots \\
\left|B^{(n)}\right|=\left(\begin{array}{c}
s \\
0
\end{array}\right)-\left(\Delta^{n} p\right)(s),
\end{array}\right.
$$

whose solution is

$$
\left|B^{(i)}\right|=\sum_{k=i}^{n}\left|B^{(k)}\right|-\sum_{k=i+1}^{n}\left|B^{(k)}\right|=\left(\begin{array}{c}
n+s-i-1 \\
n-i
\end{array}\right)-\left(\Delta^{i} p\right)(s)+\left(\Delta^{i+1} p\right)(s), \quad i<n,
$$

and $\left|B^{(n)}\right|=1$ (recall that $\left(\Delta^{i} p\right)(t) \equiv 0$ for $i>\operatorname{deg} p(t)$ and $\left.\operatorname{deg} p(t)<n\right)$. Let us call the growth vector of $p(t)$ in degree $s$ the solution of the linear system (4) and let us denote it by $\mathrm{gv}_{s}(p(t))$. 

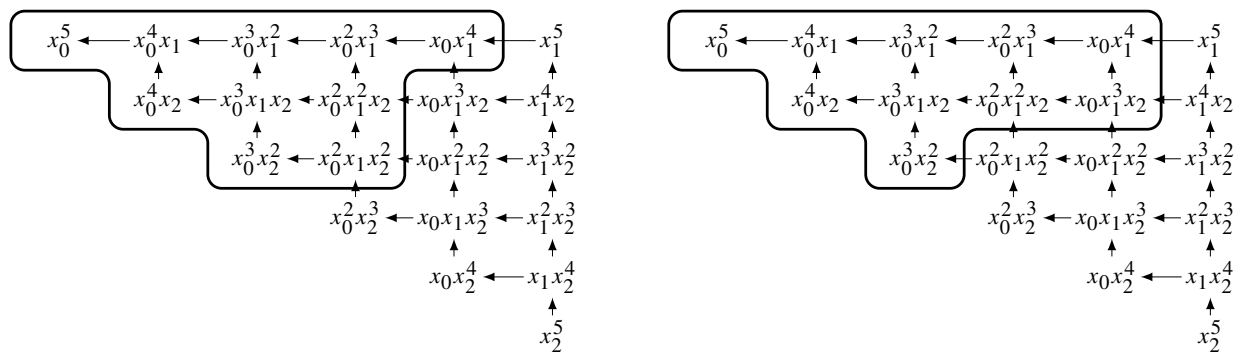

Figure 2. Borel sets in $\mathbb{T}_{2,5}$ corresponding to the saturated strongly stable ideals $\left(x_{0}^{3}, x_{0}^{2} x_{1}, x_{0} x_{1}^{4}\right)$ (on the left) and $\left(x_{0}^{3}, x_{0}^{2} x_{1}^{2}, x_{0} x_{1}^{3}\right)$ (on the right) in 3 variables with Hilbert polynomial $t+6$ and regularity at most 5 .

Proposition 3.3 (cf. [Lella 2012, Theorem 3.3]). Let $p(t)$ be a Hilbert polynomial. There is a bijective map

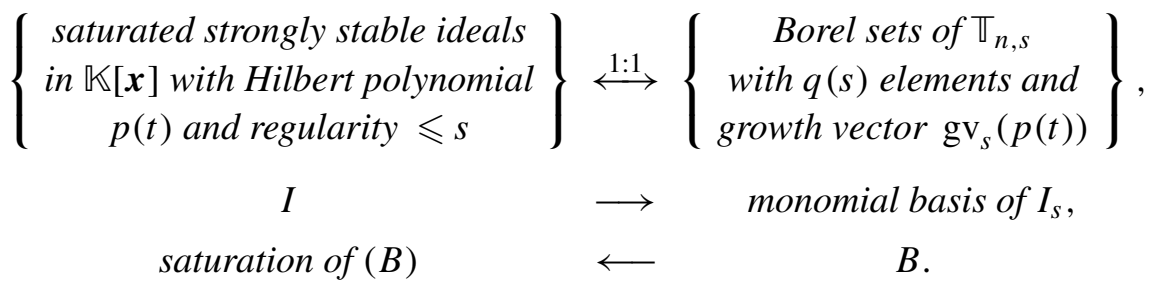

In order to determine the Borel sets of Proposition 3.3, we use a recursive algorithm based on Proposition 1.2(iii). Indeed, if $I \subset \mathbb{K}\left[x_{0}, \ldots, x_{n}\right]$ is a strongly stable ideal with Hilbert polynomial $p(t)$ and $B$ is the associated Borel set in $\mathbb{T}_{n, s}$, then the subset $B^{\prime}=\left\{x^{\alpha} \in B \mid \min x^{\alpha}>n\right\} \subset B$ is a Borel set in $\mathbb{T}_{n-1, s}$ corresponding to the strongly stable ideal $I^{\prime}=\left(x_{n}, I\right) \cap \mathbb{K}\left[x_{0}, \ldots, x_{n-1}\right] \subset \mathbb{K}\left[x_{0}, \ldots, x_{n-1}\right]$ with Hilbert polynomial $(\Delta p)(t)$.

Example 3.4. We want to determine the set of strongly stable ideals in the polynomial ring $\mathbb{K}\left[x_{0}, x_{1}, x_{2}\right]$ with regularity at most 5 defining schemes with Hilbert polynomial $p(t)=t+6$. The Gotzmann number of $p(t)$ is 6 and its growth vector in degree 5 is $\operatorname{gv}_{5}(t+6)=(5,4,1)$. We start considering the set of strongly stable ideals in $\mathbb{K}\left[x_{0}, x_{1}\right]$ with Hilbert polynomial $\Delta p(t)=1$ and regularity at most 5 corresponding to Borel sets with growth vector $\operatorname{gv}_{5}(\Delta p(t))=(4,1)$. There is a unique Borel set

$$
B^{\prime}=\left\{x_{0}^{5}, x_{0}^{4} x_{1}, x_{0}^{3} x_{1}^{2}, x_{0}^{2} x_{1}^{3}, x_{0} x_{1}^{4}\right\} .
$$

Since $x_{1}^{5}$ is not contained in $B^{\prime}$, a Borel set $B \subset \mathbb{T}_{2,5}$ with growth vector $(5,4,1)$ does not contain monomials obtained from $x_{1}^{5}$ by applying decreasing elementary moves, i.e., $x_{1}^{4} x_{2}, x_{1}^{3} x_{2}^{2}, x_{1}^{2} x_{2}^{3}, x_{1} x_{2}^{4}$ and $x_{2}^{5}$. Hence, we need to select five monomials divisible by both $x_{0}$ and $x_{2}$ producing a set closed by increasing elementary moves (see Figure 2). 
Our package provides the method stronglyStableIdeals to compute the set of strongly stable ideals of a given polynomial ring with fixed Hilbert polynomial and bounded regularity.

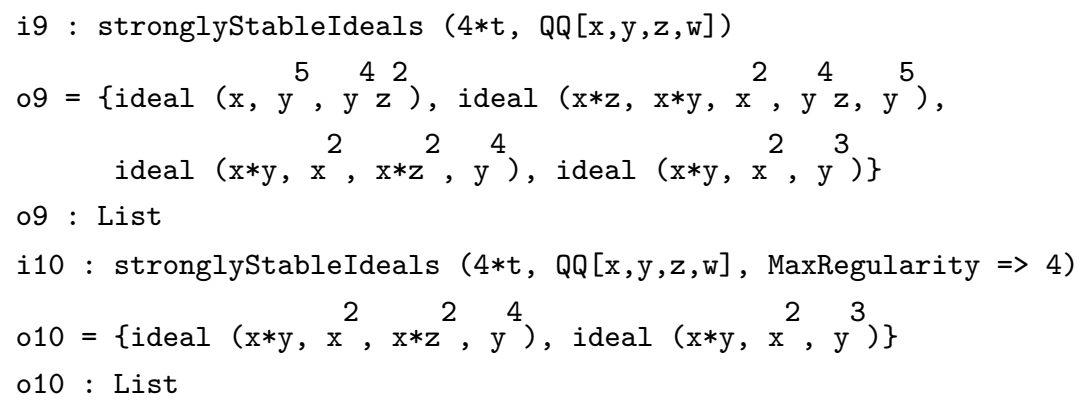

4. Segment ideals. The transitive closure of the order relation

$$
x^{\alpha}>_{B} x^{\beta} \Longleftrightarrow x^{\beta}=\mathrm{e}_{i}^{-}\left(x^{\alpha}\right)
$$

induces a partial order on the set of monomials of any degree called the Borel order. Every graded term ordering is a refinement of this partial order. Since a Borel set $B$ is closed with respect to the Borel order, i.e., $x^{\alpha}>_{B} x^{\beta}, x^{\beta} \in B \Rightarrow x^{\alpha} \in B$, it is natural to ask whether there exists a term ordering $\prec$ with the same property. For instance, for the lexicographic ideal, the graded lexicographic order separates, in each degree, monomials contained in the ideal from those outside. In [Cioffi et al. 2011], several notions of segment ideals are introduced.

Definition 4.1 [Cioffi et al. 2011, Definitions 3.1 and 3.7]. A Borel set $B \subset \mathbb{T}_{n, s}$ is called a segment if there exists a term ordering $\prec$ such that $x^{\alpha} \succ x^{\beta}$, for all $x^{\alpha} \in B$ and $\boldsymbol{x}^{\boldsymbol{\beta}} \in \mathbb{T}_{n, s} \backslash B$.

Let $I \subset \mathbb{K}[\boldsymbol{x}]$ be a saturated strongly stable ideal.

(i) $I$ is called a hilb-segment if the Borel set $I \cap \mathbb{T}_{n, r}$ is a segment, where $r$ is the Gotzmann number of the Hilbert polynomial of $I$.

(ii) $I$ is called a reg-segment if the Borel set $I \cap \mathbb{T}_{n, m}$ is a segment, where $m$ is the regularity of $I$.

(iii) $I$ is called a gen-segment if there exists a term ordering $\prec$ such that $x^{\alpha} \succ x^{\beta}$ for each minimal generator $\boldsymbol{x}^{\alpha}$ of degree $s$ of $I$ and for all $\boldsymbol{x}^{\boldsymbol{\beta}} \in \mathbb{\mathbb { T }}_{n, s} \backslash I_{s}$.

These notions are very important in the construction of flat families based on properties of Gröbner bases and in general for the study of the Hilbert scheme. The StronglyStableIdeals.m2 package provides three methods for determining whether a strongly stable ideal may be some type of segment (and, in case, gives the term ordering). These methods use tools of the package gfanInterface.m2 and the term ordering is given as a weight vector. 


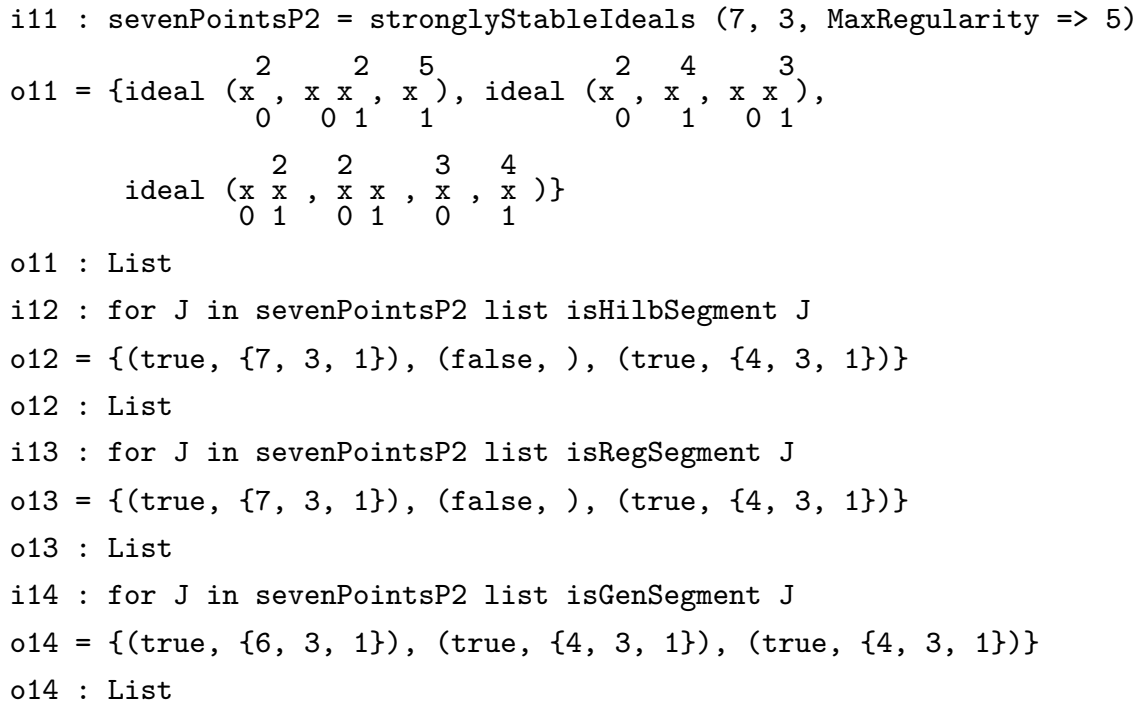

SuPPlEMENT. Version 1.1 of StronglyStableIdeals.m2 is contained in the online supplement.

\section{REFERENCES.}

[Bertone et al. 2013a] C. Bertone, F. Cioffi, P. Lella, and M. Roggero, "Upgraded methods for the effective computation of marked schemes on a strongly stable ideal", J. Symbolic Comput. 50 (2013), 263-290. MR Zbl

[Bertone et al. 2013b] C. Bertone, P. Lella, and M. Roggero, "A Borel open cover of the Hilbert scheme”, J. Symbolic Comput. 53 (2013), 119-135. MR Zbl

[Bertone et al. 2017a] C. Bertone, F. Cioffi, and M. Roggero, "Double-generic initial ideal and Hilbert scheme", Ann. Mat. Pura Appl. 196:1 (2017), 19-41. MR Zbl

[Bertone et al. 2017b] C. Bertone, F. Cioffi, and M. Roggero, "Macaulay-like marked bases", J. Algebra Appl. 16:5 (2017), 1750100, 36. MR Zbl

[Brachat et al. 2016] J. Brachat, P. Lella, B. Mourrain, and M. Roggero, "Extensors and the Hilbert scheme”, Ann. Sc. Norm. Super. Pisa Cl. Sci. 16:1 (2016), 65-96. MR Zbl

[Cioffi and Roggero 2011] F. Cioffi and M. Roggero, "Flat families by strongly stable ideals and a generalization of Gröbner bases”, J. Symbolic Comput. 46:9 (2011), 1070-1084. MR Zbl

[Cioffi et al. 2011] F. Cioffi, P. Lella, M. G. Marinari, and M. Roggero, "Segments and Hilbert schemes of points", Discrete Math. 311:20 (2011), 2238-2252. MR Zbl

[Galligo 1974] A. Galligo, “À propos du théorème de-préparation de Weierstrass”, pp. 543-579 in Fonctions de plusieurs variables complexes, edited by F. Norguet, Lecture Notes in Math. 409, Springer, 1974. MR Zbl

[Gotzmann 1978] G. Gotzmann, "Eine Bedingung für die Flachheit und das Hilbertpolynom eines graduierten Ringes”, Math. Z. 158:1 (1978), 61-70. MR Zbl

[Green 2010] M. L. Green, "Generic initial ideals", pp. 119-186 in Six lectures on commutative algebra, edited by J. Elias et al., Birkhäuser Verlag, Basel, 2010. MR 
[Lella 2012] P. Lella, "An efficient implementation of the algorithm computing the Borel-fixed points of a Hilbert scheme", pp. 242-248 in ISSAC 2012-Proceedings of the 37th International Symposium on Symbolic and Algebraic Computation, edited by J. van der Hoeven and M. van Hoeij, ACM, New York, 2012. MR Zbl

[Lella and Roggero 2011] P. Lella and M. Roggero, "Rational components of Hilbert schemes", Rend. Semin. Mat. Univ. Padova 126 (2011), 11-45. MR Zbl

[Lella and Roggero 2016] P. Lella and M. Roggero, "On the functoriality of marked families", J. Commut. Algebra 8:3 (2016), 367-410. MR Zbl

[Mall 1997] D. Mall, "Betti numbers, Castelnuovo Mumford regularity, and generalisations of Macaulay's theorem”, Comm. Algebra 25:12 (1997), 3841-3852. MR Zbl

[Moore and Nagel 2014] D. Moore and U. Nagel, "Algorithms for strongly stable ideals", Math. Comp. 83:289 (2014), 2527-2552. MR Zbl

[Reeves 1992] A. A. Reeves, Combinatorial structure on the Hilbert scheme, Ph.D. thesis, Cornell University, Ann Arbor, MI, 1992, Available at https://search.proquest.com/docview/303996863. MR

ReCEIVED: 26 Jun 2014 REvised: 22 Jun 2018 ACCEPTED: 4 Nov 2018

\section{DAVIDE Alberelli:}

davide.alberelli@gmail.com

PAOLO LELla:

paolo.lella@polimi.it

Dipartimento di Matematica, Politecnico di Milano, Milano, Italy 

Strongly stable ideals and Hilbert polynomials

Davide Alberelli and Paolo Lella

DiffAlg: a Differential algebra package

Manuel Dubinsky, César Massri, Ariel Molinuevo and Federico Quallbrunn

Matroids: a Macaulay2 package

Justin Chen

Computing quasidegrees of A-graded modules

Roberto Barrera

An algorithm for enumerating difference sets

Dylan Peifer

Hyperplane arrangements in $\mathrm{CoCoA}$

Elisa Palezzato and Michele Torielli

Numerical implicitization

Justin Chen and Joe Kileel

Random Monomial Ideals: a Macaulay2 package

Sonja Petrović, Despina Stasi and Dane Wilburne

Ben Drabkin, Eloísa Grifo, Alexandra Seceleanu and Branden Stone

The gfanlib interface in Singular and its applications

Anders Jensen, Yue Ren and Hans Schönemann 